\title{
BIODIVERSIDAD EN EL ÁREA DE INFLUENCIA DE LA ESTACIÓN PRIMATES DE COLOSÓ, SUCRE, COLOMBIA.
}

\author{
BIODIVERSITY IN THE INFLUENCES AREA IN COLOSÓ PRIMATES STATION, \\ SUCRE, COLOMBIA.
}
GALVÁN-GUEVARA, SILVIA ${ }^{1}$ Biol. SIERRA, M. IVAN ${ }^{2}$ MVZ. GÓMEZ, F. HERNANDO ${ }^{1}$ Biol. DE LA OSSA, V. JAIME ${ }^{3}$ Dr. FAJARDO-PATIÑO, ALIRIO ${ }^{4}$ M.Sc

\footnotetext{
${ }^{1}$ Universidad de Sucre, Dpto. de Biología. ${ }^{2}$ CARSUCRE. ${ }^{3}$ Universidad de Sucre, Facultad. Ciencias Agropecuarias, Grupo Biodiversidad Tropical.

${ }^{4}$ Fundación Bioma. Correspondencia: silgague@gmail.com
}

\section{Resumen}

El presente trabajo muestra de manera comparativa la presencia más notoria de las diferentes especies de fauna silvestre existentes en la zona de influencia de la Estación de Primatología del municipio de Colosó, departamento de Sucre, Colombia; así mismo de manera relacionada hace referencia a la variabilidad de hábitat presentes y que conforman una estructura ambiental compleja y altamente diversa para el bosque seco que allí se presenta.

Palabras clave: Fauna silvestre, hábitat, bosque seco, Colosó, Sucre. Colombia.

\section{Abstract}

The current paper shows in a comparative way the most noticeable presence of the different existent species of wildlife in the area of influence of the Primates Station on the municipality of Colosó, Sucre-Colombia. In the same way, in a related way it makes reference to the variability of habitat which comprises a complex and highly diverse environmental structure for the dry forest that is shown there, thus showing the importance of the socioeconomic content of the zone and its influence in the area.

Key words: Wildlife, habitat, dry forest, Colosó, Sucre. Colombia. 


\section{Introducción}

La fauna silvestre, en un sentido amplio, abarca todos los animales no domésticos; el término "vida silvestre", equivalente a wildlife en inglés, es aún más amplio (WING, 1951; SCHUERHOLZ y MANN, 1979; USHER, 1986); sin embargo, el término fauna silvestre se emplea generalmente en sentido más específico para denominar al conjunto de animales que concuerda con el uso cotidiano de esta expresión (BELTRÁN, 1966; GILES, 1978; GONDELLES et al., 1981). Las especies nativas en su conjunto constituyen la riqueza y diversidad genética de los ecosistemas y forman parte del patrimonio natural de países, regiones y del mundo (DE LA OSSA y RIAÑO, 1997; OJASTI, 2000).

El sistema de valoración que maneja la sociedad se fundamenta en el aporte o utilidad de las cosas para el hombre, es decir, en su valor tangible o económico, expresado generalmente en unidades monetarias. Este valor puede ser 1) el valor de mercado, 2) el valor directo no comercial ó 3) el valor indirecto no comercial (ONU, 1994).

A pesar de sus múltiples valores, la fauna silvestre es el más subestimado de los recursos naturales renovables, porque salvo contadas excepciones, carece de vocación comercial y no genera estadísticas comparables con los recursos pesqueros o forestales; la justa valoración económica de la fauna silvestre, sin detrimento de sus valores intangibles es vital porque las grandes decisiones políticas se fundamentan ante todo en argumentos económicos (MCNEELY, 1988; UNEP, 1993).

Esto es particularmente cierto en los países latinoamericanos que se ven en la necesidad de hacer un uso prudente de todos sus recursos, a fin de sustentar su crecimiento demográfico y económico; al mismo tiempo, es esencial que los habitantes locales sean los beneficiarios principales de los valores de la fauna silvestre, lo cual puede incentivarlos a usar este recurso de una manera sostenible (OJASTI, 2000). 
La desaparición o degradación severa de hábitat, es sin duda la causa principal del aniquilamiento progresivo de la fauna silvestre nacional, tanto que sus efectos exceden por mucho a los debidos por la caza incontrolada (PPIAS, 2005); sin embargo, en la medida que se destruyen los últimos relictos de bosques nativos, se tornan difíciles las condiciones de supervivencia de las diferentes especies de fauna silvestre, debido a la falta de una adecuada oferta de alimentos y a la garantía de un refugio seguro (OVIEDO, 2008), por fragmentación reproductiva, aislamiento genético y pérdida de plasticidad adaptativa (PRIMACK, 1998).

Se aborda de manera sistemática el reconocimiento de las principales especies de fauna silvestre que se encuentran presentes en los Montes de María, con especial referencia a la zona de influencia de la Estación Primatológica existente en Colosó, Sucre, Colombia; contrastando los registros con las referencia especificas halladas en trabajos anteriores, buscando exponer un marco referencial que sirva de apoyo a futuras investigaciones relacionadas.

\section{Caracterización general}

El departamento de Sucre se encuentra localizado en la planicie costera Caribe al norte de las cordilleras Central y Occidental, entre los $10^{\circ} 9^{\prime}$ y los $8^{\circ} 17^{\prime}$ Norte y los $74^{\circ} 32^{\prime}$ y $75^{\circ} 42^{\prime}$ Oeste, con una superficie de 10.364 Km². La jurisdicción de CARSUCRE cubre la parte Norte del Departamento, presenta una faja de colinas, que corresponden a la Serranía de San Jacinto o Montes de María (Fig. 1). Entre estas colinas y la depresión del río San Jorge se localizan sabanas antropogénicas de topografía ondulada (AGUILERA, 2005).

El clima característico de la subregión es cálido y seco; dado que no existe un relieve montañoso que incida significativamente sobre la temperatura, las variaciones máximas y mínimas no superan los $3^{\circ} \mathrm{C}$, de tal forma que los diversos relieves del área jurisdiccional corresponden al piso térmico cálido con temperaturas que varían de los $25.5^{\circ} \mathrm{C}$ a los $28.7^{\circ} \mathrm{C}$. Los meses más calurosos son enero, febrero, marzo y julio. La radiación promedio está entre 2.600 a $3100 \mathrm{cal} / \mathrm{cm}^{2} / \mathrm{semana}$ (IGAC, 1969; AGUILERA, 2005). 


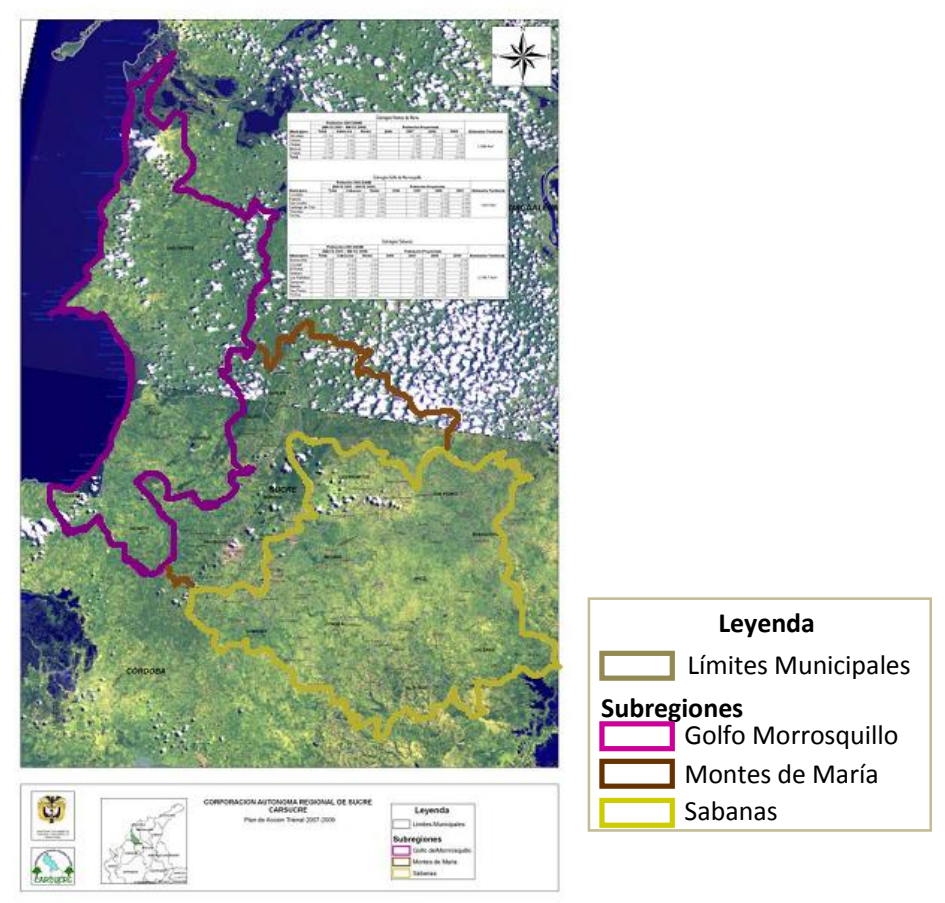

Figura.1. Localización de las Subregiones de CARSUCRE (Fuente: PAT-Carsucre, 20072009).

Las condiciones de precipitación de lluvias de la zona de jurisdicción de la Corporación Autónoma Regional de Sucre (CARSUCRE) se encuentran en un rango de 896.0 a $1233.1 \mathrm{~mm}$ de precipitación promedio anual. La humedad relativa está promediando el $83.5 \%$ anual y la evaporación media 1.572 mm/año. (IGAC, 1969.; AGUILERA, 2005).

\section{Subregión Montes de María}

Ecológicamente es una zona estratégica para el Departamento y es una geoforma que aparentemente hace parte de las estribaciones septentrionales de la serranía de San Jerónimo que presenta una depresión hipsométrica en el Noreste de Córdoba para ir ganando niveles altitudinales en rumbo Noreste, hasta alcanzar su máxima altura de $800 \mathrm{msnm}$ en el Cerro Maco en el departamento de Bolívar; en Sucre la mayor altura alcanzada son los 600 msnm en la loma La Pita en límites con Bolívar. Esta geoforma se origino por 
eventos tectónicos del Mioceno durante los cuales levantamientos orogénicos generaron el anticlinal de San Jacinto que dio lugar a los Montes de María y la Serranía de San Jacinto y que en sus estratos más antiguos se evidencia su origen coralino.

Geológicamente está conformada por rocas del Cretáceo Superior y mantos de arenas, arcillas y calizas del Eoceno-Mioceno (IGAC, 1969). Se localizada en la zona Noreste del Departamento constituida por los municipios de Colosó, Chalán, Morroa, Ovejas y Sincelejo; posee una superficie de $1.104 \mathrm{Km}^{2}$ que corresponde al $10.6 \%$ del área departamental. Desde el punto de vista biótico es la región más compleja y de mayor interés por sus potencialidades ecoturísticas y de riqueza en recursos genéticos, importantes para el desarrollo de actividades de bioprospección y biocomercio (IGAC, 1969; MINISTERIO DEL AMBIENTE, 2002; MMA - DCBIO, 2004).

En esta subregión se encuentra localizada la Estación Primatológica de CARSUCRE, en las estribaciones de la serranía de Coraza-Montes de María, situada en el municipio de Colosó ( $9^{\circ} 29^{\prime}$ Noreste y $75^{\circ} 21^{\prime}$ Oeste), en la planicie del litoral Caribe colombiana y que localmente recibe el nombre de Montes de María; caracterizada por bosques higrotropofiticos, subhigrofíticos y freatofíticos. Los elementos florísticos más conspicuos son Anacardium excelsum, Brownea macrophylla, Enterolobium cyclocarpum, Bursera simaruba, Pachira quinata, Manilkara zapota, Pseudobombax septenatum, Uribea tamarindoides, Hura crepitans, Sterculia apetala y Spondias Bombin, entre otras (CUERVO et al.,1986a).

\section{Bosques característicos de la zona de influencia de la Estación Primatológica}

La subregión Montes de María en la localidad de Colosó en alrededores de la Estación de Primates a nivel de vegetación ha sido estudiada en varias oportunidades y pueden distinguirse dos formaciones (BARBOSA et al., 1988). 
1. Vegetación de ladera que corresponde a los bosques de tipo higrotropofítico calcícolas, desarrollados en fuertes pendientes con afloramientos de rocas calcáreas de origen coralino, el dosel se encuentra alrededor de los $25 \mathrm{~m}$ y hay predominancia de especies caducifolias. El estrato arbóreo es heterogéneo con la presencia de Aspidosperma polyneuron, Brosimun utile, Bursera simaruba, Pseudobombax septenatum, Hura crepitans y Uribea tamarindoides, entre otras. El estrato arbustivo muestra a Pachira quinata, Brosimum utile, Pithecellobium dulce y Triplaris americana, en el estrato herbáceo se encuentra Anthurium sp., Cnidosculus tubulosus y Jatropha sp.

2. Vegetación riparia, que corresponde a los bosques de galería desarrollados en los márgenes de los arroyos, en suelos franco arcillosos, ricos en nutrientes y buena disponibilidad de recurso hídrico. La vegetación se caracteriza por presentar en su estrato arbóreo elementos florísticos dominantes como Anacardium excelsum asociados a Brownea macrophylla, Enterolobium cyclocarpum, Gyrocarpus americanus, Hura crepitans, Lecythis magdalenica, Spondias mombin, Sterculia apetala, Manilkara zapota, Ficus sp y Albizzia sp. En el estrato arbustivo se encuentran Brownea macrophylla, Genipa americana, Inga spectabilis, Ficus sp., Pithecellobium dulce, Garcia nutans, Capparis odoratissima y Erythrina sp. En el otro estrato subordinado o sunarbustivo se localizan Bactris major, Acalypha sp., Gustavia sp., Randia sp., Heliconia sp., Piper marginatum y Aphelandra sp.

Para una mejor comprensión del sistema planteado se muestra el perfil de vegetación registrado por CUERVO et al. (1986), del levantamiento realizado en los alrededores de la Estación de Primates, sector La Esmeralda (Fig. 3). 


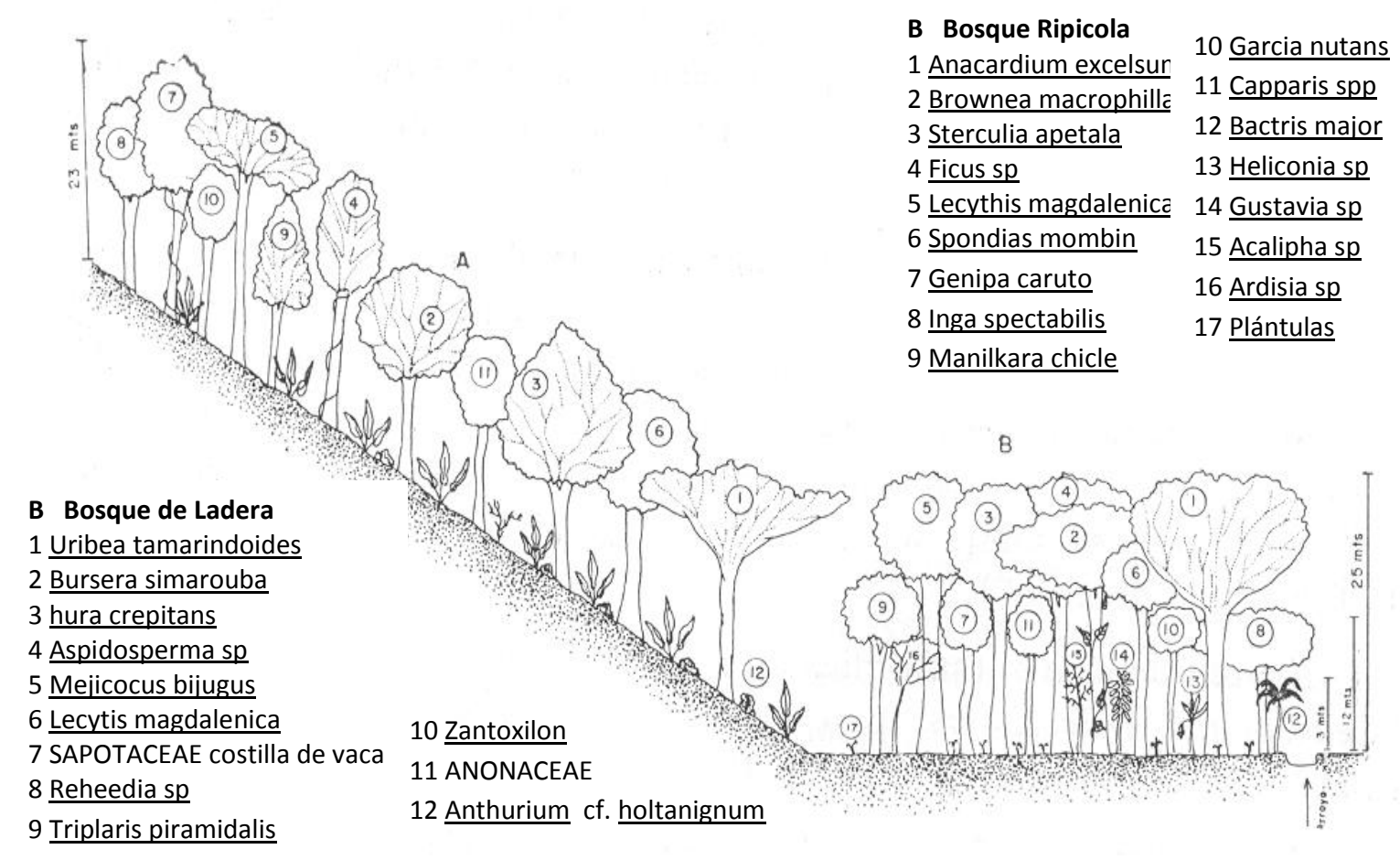

Figura. 3 Perfil Diagramático de vegetación del Bosque de ladera y ripícola de Colosó "La Esmeralda" (Fuente: CUERVO et al., 1986)

\section{Aspectos Bióticos}

\section{Evaluación de información secundaria y comprobación de campo}

Se realizó la búsqueda de información secundaria básica, para lo cual se revisó la literatura disponible en la Corporación Autónoma Regional de Sucre "CARSUCRE", Biblioteca Banco de la República, Biblioteca Universidad de Sucre, Bibliotecas personales y literatura disponible en la red; se evaluó la información obtenida y se establecieron los listados por grupo taxonómico de las especies de fauna silvestre perteneciente a los vertebrados y que se tuvieran como registradas para el área de estudio (DAHL, 1971; FROST, 1985; HILTY y BROWN, 1986; EISEMBERG, 1989; SÁNCHEZ et al., 1992; RUÍZ et al., 1996; MUÑOZ-ARANGO, 2001; CASTAÑO-MORA, 2002; RUEDA- 
ALMONACID et al., 2004; RODRÍGUEZ-MAHECHA et al., 2006) (Tablas 1 a 5).

\section{Peces}

Tabla 1. Listado de peces citados para la zona de estudio

\begin{tabular}{|c|c|c|}
\hline Familia & N. Científico & N. Común \\
\hline PIMELOLIDAE & Pimelodella chagresi & picalón \\
\hline \multirow{2}{*}{ CALLICHTHYDADE } & $\begin{array}{c}\text { Hoplosternum thoracatun } \\
\text { magdalenae }\end{array}$ & chipe \\
\hline \multirow{4}{*}{ LORICARIDAE } & Cochliodon hondae & $\begin{array}{c}\text { coroncoro } \\
\text { cucho }\end{array}$ \\
\cline { 2 - 3 } & Loricaria filamentosa & alcalde \\
\hline \multirow{4}{*}{ CHARACIDAE } & Curimata magdalenae & yalúa \\
\cline { 2 - 3 } & Creagrutus nigrostigmatus & chispa \\
\cline { 2 - 3 } & Hoplias malabaricus & moncholo \\
\cline { 2 - 3 } & Saccoderma falcatum & brincona \\
\cline { 2 - 3 } & Astyanax magdalenae & sardina \\
\cline { 2 - 3 } & Astyanax foscuatus & sardina coliroja \\
\hline SYNGNATHIDAE & Pseudafallus mindii & palito \\
\hline CYPRINODONTIDAE & Austrofundulus myersi & azulejo \\
\hline POECILIIDAE & Mollienisia caucana & pipon \\
\hline
\end{tabular}

\section{Herpetofauna}

\section{Anfibios}

Tabla 2. Listado de anfibios citados para la zona de estudio.

\begin{tabular}{|c|c|c|}
\hline Familia & N. Científico & N. Común \\
\hline CAECILIDAE & Caecilia subnigricans & ciega \\
\hline TYPHLONECTIDAE & Typhlonectes natans & anguila \\
\hline PLETHODONTIDAE & Bolitoglossa biseriata & salamandra \\
\hline \multirow{3}{*}{ BUFONIDAE } & Bufo (Chaunus) granulosus & sapito \\
\cline { 2 - 3 } & Bufo (Chaunus) haematiticus & sapo \\
\cline { 2 - 3 } & Bufo (Chaunus) marinus & sapo \\
\hline \multirow{2}{*}{ CENTROLENIDAE } & Hyalinobatrachium colymbiphyllum & rana de cristal \\
\cline { 2 - 3 } & Hyalinobatrachium fleishmanni & rana de cristal \\
\hline
\end{tabular}




\begin{tabular}{|c|c|c|}
\hline DENDROBATIDAE & Dendrobates truncatus & rana venenosa \\
\hline \multirow{5}{*}{ HYLIDAE } & Hyla boans & rana \\
\hline & Hyla ebraccata & ranita \\
\hline & Hyla microcephala & rana \\
\hline & Hyla pugnax & rana platanera \\
\hline & Hyla vigilans & desconocido \\
\hline \multirow{7}{*}{ HYLIDAE } & Phrynohyas venulosa & rana \\
\hline & Phyllomedusa venusta & desconocido \\
\hline & Scinax boulengeri & desconocido \\
\hline & Scinax elaeochora & desconocido \\
\hline & Scinax rubra & desconocido \\
\hline & Smilisca phaeota & desconocido \\
\hline & Smilisca sila & desconocido \\
\hline \multirow{12}{*}{ LEPTODACTYLIDAE } & Ceratophrys calcarata & sapo cuerno \\
\hline & Eleutherodactylus bufoniformis & desconocido \\
\hline & Eleutherodactylus gaigei & desconocido \\
\hline & Eleutherodactylus raniformis & desconocido \\
\hline & Eleutherodactylus sp & desconocido \\
\hline & Leptodactylus bolivianus & desconocido \\
\hline & Leptodactylus fragilis & desconocido \\
\hline & Leptodactylus fuscus & rana picuda \\
\hline & Leptodactylus pentadactylus & desconocido \\
\hline & Physalaemus pustulosus & vaquero \\
\hline & Pleuroderma brachyops & desconocido \\
\hline & Pseudopaludicola pusilla & desconocido \\
\hline \multirow{2}{*}{ MYCROHYLYDAE } & Chiasmocleis panamensis & desconocido \\
\hline & Relictivomer pearsei & rana picuda \\
\hline PSEUDIDAE & Pseudis paradoxa & rana de pantano \\
\hline
\end{tabular}

\section{Reptiles}

Tabla 3. Listado de reptiles citados para la zona de estudio

\begin{tabular}{|c|c|c|}
\hline Familia & N. Científico & N. Común \\
\hline IGUANIDAE & Anolis auratus & lagartija \\
\hline
\end{tabular}




\begin{tabular}{|c|c|c|}
\hline \multirow{6}{*}{ IGUANIDAE } & Anolis malkini & lagartija \\
\hline & Anolis tropidogaster & lagartija \\
\hline & Anolis vittigerus & lagartija \\
\hline & Basiliscus basiliscus & pasa arroyos \\
\hline & Corytophanes cristatus & desconocido \\
\hline & Iguana iguana & iguana \\
\hline \multirow{3}{*}{ GEKKONIDAE } & Gonatodes albogularis & desconocido \\
\hline & Hemidactylus brooki & salamanquejas \\
\hline & Thecadctylus rapicauda & salamanquejas \\
\hline GYMNOPHTHALMIDAE & Leposoma rugiceps & desconocido \\
\hline SCINIDAE & Mabuya mabouya & lisa \\
\hline \multirow{4}{*}{ TEIIDAE } & Ameiva ameiva & lobito \\
\hline & Ameiva festiva & lobito \\
\hline & Cnemipophorus lemniscatus & lobito \\
\hline & Tupinanambis teguixin & lobo pollero \\
\hline ANOMALEPIDIDAE & Liotyphlops albirostris & ciega \\
\hline LEPTOTYPHLOPIDAE & Leptotyphlops macrolepis & ciega \\
\hline \multirow{3}{*}{ BOIDAE } & Boa constrictor & boa \\
\hline & Corallus ruschenbergerii & mataboga \\
\hline & Epicrates cenchria maurus & $\begin{array}{c}\text { pudridora, boa } \\
\text { tornasol }\end{array}$ \\
\hline \multirow{11}{*}{ COLUBRIDAE } & Chironius carinatus & $\begin{array}{c}\text { amarillo, lomo de } \\
\text { machete }\end{array}$ \\
\hline & Clelia clelia & candelilla \\
\hline & Dendrophidium bivittatus & guarda camino \\
\hline & Dendrophidium percarinatus & cazadora \\
\hline & Helicops danieli & mapaná de agua \\
\hline & Imantodes cenchoa & bejuquilla \\
\hline & Leptodeira septentrionalis & mapaná falsa \\
\hline & $\begin{array}{c}\text { Leptophis ahaetulla } \\
\text { occidentales }\end{array}$ & bejuca \\
\hline & Liophis lineatus & guarda camino \\
\hline & Liophis melanotus & guarda camino \\
\hline & Mastygodrias boddaerti & cazadora \\
\hline
\end{tabular}




\begin{tabular}{|c|c|c|}
\hline \multirow{4}{*}{ COLUBRIDAE } & Mastygodrias pleei & cazadora \\
\hline & Ninia atrata & desconocido \\
\hline & Oxybelis aeneus & bejuquilla \\
\hline & Oxirhopus petola & coral falsa \\
\hline \multirow{8}{*}{ COLUBRIDAE } & Pseudoboa neuwiedii & $\begin{array}{c}\text { candelilla roja, coral } \\
\text { macho }\end{array}$ \\
\hline & $\begin{array}{c}\text { Pseustes poecilonotus } \\
\text { polylepis }\end{array}$ & desconocido \\
\hline & Pseustes shropshirei & cazadora \\
\hline & Sibon nebulata & desconocido \\
\hline & Spilotes pullatus & toche \\
\hline & Stenorrhina degenhardtii & desconocido \\
\hline & Thamnodynastes pallidus & guarda camino \\
\hline & Urotheca euryzonus & coral falsa \\
\hline \multirow{2}{*}{ ELAPIDAE } & Micrurus dissoleucus & coral \\
\hline & Micrurus sp & coral \\
\hline \multirow{3}{*}{ VIPERIDAE } & Bothriechis schlegeli & dormilona \\
\hline & Bothrops asper & mapaná negra \\
\hline & Porthidium nasutum & patoco \\
\hline EMYDIDAE & Trachemys scripta & Icotea* \\
\hline KINOSTERNIDAE & Kinosternon scorpioides & Tacán** \\
\hline TESTUDINIDAE & Geochelone carbonaria & Morrocoy*** \\
\hline ALLIGATORIDAE & Caiman crocodilus fuscus & Babilla* \\
\hline
\end{tabular}

* En jagüeyes vecinos típicos de áreas agropecuarias, no se obtuvo información sobre su origen inicial. ${ }^{* *}$ En zonas abiertas aledañas al bosque preferencialmente en rastrojos. ${ }^{* * *}$ Solo en las viviendas de los pobladores, los crían como animales de estimación y les adjudican popularmente mitos y leyendas de buena suerte. 


\section{Ornitofauna}

Tabla 4. Listado de Aves citados para la zona de estudio

\begin{tabular}{|c|c|c|}
\hline Familia & N. Científico & N. Común \\
\hline \multirow{2}{*}{ TINAMIDAE } & Cripturellus soui & tinamú \\
\hline & Cripturellus eritheopus & tinamú rojo \\
\hline \multirow{5}{*}{ ARDEIDAE } & Butorides striatus & garcita rayada \\
\hline & Bubulcus ibis & garcita del ganado \\
\hline & Philerodius pileatus & garza crestada \\
\hline & Nycticorax nycticorax & guaco común \\
\hline & Tigrisoma lineatum & vaco común \\
\hline \multirow{2}{*}{ THRESKIORNITIDAE } & Mesembrinibis cayennensis & ibis verde \\
\hline & Ajaia ajaia & espatula \\
\hline \multirow{2}{*}{ ANATIDAE } & Dendrocygna viduata & viudita \\
\hline & Dendrocygna autumnalis & pisingo \\
\hline \multirow{3}{*}{ CATHARTIDAE } & Cathartes aura & guala \\
\hline & Coragyps atratus & golero \\
\hline & Sarcorramphus papa & rey golero \\
\hline PANDIONIDAE & Pandion haliaetus & aguila pescadora \\
\hline \multirow{10}{*}{ ACCIPITRIDAE } & Leptodon cayannensis & aguila cabeza gris \\
\hline & Gampsonyx swainsonii & aguila enana \\
\hline & Rostrhamus sociabilis & caracolero \\
\hline & Geranospiza caerulescens & aguila zancona \\
\hline & Accipiter bicolor & halcon \\
\hline & Buteogallus urubitinga & bebe humo \\
\hline & Heterospizia meridionalis & aguila \\
\hline & Parabuteo unicinctus & agila \\
\hline & Buteo nitidus & gavilan \\
\hline & Buteo magnirostris & gavilan \\
\hline \multirow{4}{*}{ FALCONIDAE } & Daptirus Sp & halcon \\
\hline & Polyborus plancus & caracara \\
\hline & Milvago chimachima & pigua \\
\hline & Herpetotheres cachinnans & halcon culebrero \\
\hline
\end{tabular}




\begin{tabular}{|c|c|c|}
\hline \multirow[b]{2}{*}{ FALCONIDAE } & Falco sparverus & halcon \\
\hline & Falco peregrinus & halcon \\
\hline \multirow{3}{*}{ CRACIDAE } & Ortallis garrula & guacharaca \\
\hline & Penelope purpurascens & pava de monte \\
\hline & Crax alberti & paujil \\
\hline PHASIANIDAE & Colinus cristatus & perdiz \\
\hline \multirow{2}{*}{ RALLIDAE } & Aramis $s p$ & chilacoa \\
\hline & Porphyrio martinica & polla azul-tanga \\
\hline JACANIDAE & Jacana jacana & gallito de cienaga \\
\hline CHARADRIIDAE & Vanellus chilenis & galan \\
\hline \multirow{4}{*}{ COLUMBIDAE } & Columba cayennensis & guarumera \\
\hline & Colombina minuta & tortolita \\
\hline & Columbina talpacoti & tierrerita \\
\hline & Claravis pretiosa & tortola \\
\hline COLUMBIDAE & Leptotila verreauxi & rabiblanca \\
\hline \multirow{7}{*}{ PSITTACIDAE } & Ara arauna & gonzalo \\
\hline & Ara macao & guacamaya \\
\hline & Aratinga pertinax & cotorra \\
\hline & Brotogeris juglaris & perico \\
\hline & Pionus menstruus & periquito \\
\hline & Amazona ochrocephala & lora \\
\hline & Amazona amazonica & manglera \\
\hline \multirow{4}{*}{ CUCULIDAE } & Piaya cayana & cuco \\
\hline & Crotophaga ani & chingola \\
\hline & Crotophaga major & chingola grande \\
\hline & Crotophaga sulcirostris & chingola azulada \\
\hline TYTONIDAE & Tyto alba & lechuza \\
\hline \multirow{3}{*}{ STRIGIDAE } & Otus choliba & currucutú \\
\hline & Bubo virginianus & buho real \\
\hline & Pulsatrix perspicillata & buho de anteojos \\
\hline \multirow{2}{*}{ CAPRINULGIDAE } & Chordeiles acutipennis & chotacabras \\
\hline & Nyctidromus albicolis & gallina ciega \\
\hline \multirow[b]{2}{*}{ TROCHILADAE } & Glaucis hirsuta & colibri ermitaño \\
\hline & Phaetornis anthophilus & colibri ermitaño negro \\
\hline
\end{tabular}




\begin{tabular}{|c|c|c|}
\hline \multirow{6}{*}{ TROCHILADAE } & Anthracothorax nigricolis & chupaflor \\
\hline & Chlorostilbon gibsoni & colibri picorojo \\
\hline & Thaluramia $s p$ & ninfa colibri \\
\hline & Damophila julie & pechinerde \\
\hline & Lepidopyga goudoti & colibri de goudot \\
\hline & Amazilia sp & colibri \\
\hline TROGONIDAE & Trogon sp & trogon \\
\hline \multirow{2}{*}{ ALCENIDAE } & Ceryle torguata & martin pescador \\
\hline & Chloroceryle Sp & martin pescador \\
\hline MOMOTIDAE & Momotus momota & barranquero \\
\hline GALBULIDAE & Galbula $S p$ & jacamar \\
\hline \multirow{2}{*}{ BUCCONIDAE } & Notharchus macrorhynchas & bobo \\
\hline & Hypnellas ruficollis & bobo jabado \\
\hline \multirow{3}{*}{ PICIDAE } & Chrysoptilus punctigula & carpintero buchipecoso \\
\hline & Piculus sp & carpintero \\
\hline & Dryocopus lineatus & carpintero real \\
\hline \multirow[t]{2}{*}{ PICIDAE } & Melanerpes rubricapillus & carpintero jabado \\
\hline & Campephilus melanoleucos & carpintero \\
\hline \multirow{4}{*}{ DENDROCOLAPTIDAE } & Dendrocincla fuliginosa & trepador pardo \\
\hline & Dendrocolaptes certhia & trepador rallado \\
\hline & Xiphorhynchus guttatus & trepador \\
\hline & Lepidocolaptes souleyetii & trepador \\
\hline \multirow{3}{*}{ FURNARIIDAE } & Furnarius leucopus & hornero patiamarillo \\
\hline & Synallaxis $s p$ & rastrojero \\
\hline & Xenops minutus & rastrojero \\
\hline \multirow{3}{*}{ FORNICARIDAE } & Sakesphorus canadensis & batará copeton \\
\hline & Formicivora grisea & hormiguero negro \\
\hline & Formicarius nigricapillas & hormiguero negro \\
\hline \multirow{2}{*}{ PIPRIDAE } & Monacus monacus & saltarincito \\
\hline & Schiftormis turdinus & saltarin \\
\hline COTINGIDAE & Pachyramphus cinnamoneus & cabezon \\
\hline \multirow{3}{*}{ TYRANNIDAE } & Camptostoma obselotum & tiranudo \\
\hline & Milopagis gaimardii & elaenia \\
\hline & Elaenia flavogaster & elaenia \\
\hline
\end{tabular}




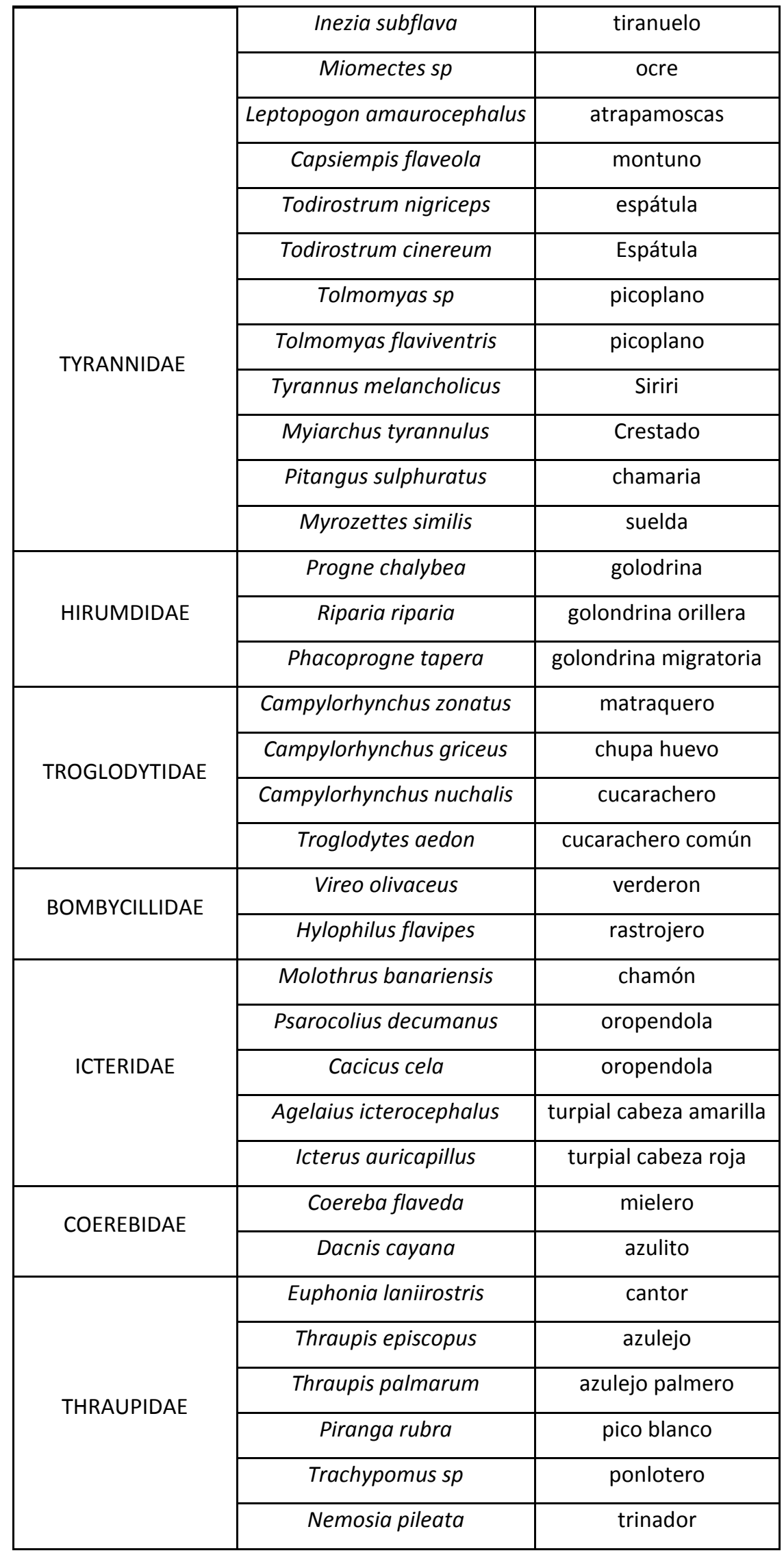




\begin{tabular}{|c|c|c|}
\hline \multirow{4}{*}{ FRINGILLIDAE } & Saltator maximus & saltarin \\
\cline { 2 - 3 } & Oryzoborus crassirostris & yolofo \\
\cline { 2 - 3 } & Sporophila nigricolis & espiguero \\
\cline { 2 - 3 } & Sporophila minuta & meriño \\
\cline { 2 - 3 } & volantina jacarina & mochuelo \\
\cline { 2 - 3 } & Sicalis flaveola & canario \\
\hline
\end{tabular}

\section{Mastofauna}

Tabla 5. Listado de Mamíferos citados para la zona de estudio

\begin{tabular}{|c|c|c|}
\hline Familia & N. Científico & N. Común \\
\hline \multirow{4}{*}{ DIDELPHIDAE } & Caluromys derbianus & zorrita lanuda \\
\hline & Chironectes minimus & comadreja \\
\hline & Didelphis marsupialis & zorra chucha \\
\hline & Marmosa robinsoni & marmota \\
\hline MYRMECOPHAGIDAE & Tamandua mexicana & hormiguero \\
\hline \multirow{2}{*}{ BRADIPODIDAE } & Bradypus variegatus & perezoso tres dedos \\
\hline & Choleopus hoffmanni & perezoso dos dedos \\
\hline \multirow{2}{*}{ DASYPODIDAE } & Dasypus novemcinctus & armadillo \\
\hline & Cabassous Centralis & mojan \\
\hline \multirow{3}{*}{ EMBALLONURIDAE } & Rhynchonycteris naso & murcielago de trompa \\
\hline & Sacopterix bilineata & murcielago rayado \\
\hline & Diclidurus albus & murcielago fantasma \\
\hline NOCTILIONIDAE & Noctilio albiventris & murcielago pezcador \\
\hline \multirow{2}{*}{ MORMOOPIDAE } & Pteronotus parnelli & murcielago bigotudo \\
\hline & Pteronotus davyi & murcielago peludo \\
\hline \multirow{7}{*}{ PHYLLOSTOMIDAE } & Mycronicteris hirsuta & murcielago pequeño \\
\hline & Lonchorhina aurita & murcielago espada \\
\hline & Tonatia brasiliense & murcielago oreja redonda \\
\hline & Tonatia silvicola & murcielago oreja redonda \\
\hline & Phyllostomus hastatus & murcielago nariz \\
\hline & Vampyrum spectrum & murcielago vampiro \\
\hline & Glossophaga soricina & murcielago nectario \\
\hline
\end{tabular}




\begin{tabular}{|c|c|c|}
\hline \multirow{11}{*}{ PHYLLOSTOMIDAE } & Lonchophila mordax & murcielago marron \\
\hline & Carollia brevicauda & murcielago colicorto \\
\hline & Carollia perspecilata & murcielago colicorto \\
\hline & Sturnira lilium & murcielago amarillo \\
\hline & Uroderma bilobatum & murcielago amarillo \\
\hline & Vampyrops helleri & murcielago lineado \\
\hline & Vampyrops vitattus & murcielago lineado \\
\hline & Artibeos jamaicensis & murcielago frutero \\
\hline & Artibeos cinereos & murcielago frutero \\
\hline & Artibeos litoratus & murcielago frutero \\
\hline & Desmodus rotundus & vampiro \\
\hline \multirow{2}{*}{ VESPERTILIOMIDAE } & Myotis albescens & murcielago marroncito \\
\hline & Eptesicus brasiliensis & oreja de raton \\
\hline MOLOSSIDAE & Molosops planirostris & cola de ratón \\
\hline CALLITRICIDAE & Saguinus oedipus & titi cabeza blanca \\
\hline \multirow{3}{*}{ CEBIDAE } & Aotus lemurinus & martica \\
\hline & Cebus capucinus & cariblanca \\
\hline & Alouatta seniculus & mono aullador \\
\hline CEBIDAE & Ateles paniscus & mono araña \\
\hline \multirow{2}{*}{ CANIDAE } & Cerdocyon thous & zorra perro \\
\hline & Speothos venaticus & perro de monte \\
\hline \multirow{4}{*}{ PROCYONIDAE } & Procyon cancrivorus & mapache \\
\hline & Nasua nasua & cusumbo \\
\hline & Potos flavus & perrito de monte \\
\hline & Eira barbara & taira \\
\hline \multirow{4}{*}{ FELIDAE } & Leopardus tigrina & gato de monte \\
\hline & Leopardus pardalis & tigrillo \\
\hline & Leopardus wiiedi & tigrillo \\
\hline & Herpairulus yaguaroundi & mojoso \\
\hline TAYASSUIDAE & Tayassu tajacu & pueerco monte \\
\hline CERVIDAE & Mazama americana & venado \\
\hline SCIURIDAE & Sciurus granatensis & ardilla \\
\hline \multirow[b]{2}{*}{ MURIDAE } & Oryzomis bicolor & raton arrocero \\
\hline & Rheomys sp & raton pezcador \\
\hline
\end{tabular}




\begin{tabular}{|c|c|c|}
\hline MURIDAE & Zigodontomys brevicaudata & raton cola corta \\
\hline \multirow{2}{*}{ MURINAE } & Rathus rathus & rata negra \\
\cline { 2 - 3 } & Mus musculus & raton casero \\
\hline AGOUTIDAE & Agouti paca & guartinaja \\
\hline DASYPROCTIDAE & Dasyprocta punctata & ñeque \\
\hline ECHYMYIDAE & Proechimys canicollis & rata espinosa \\
\hline LEPORIDAE & Silvilagus floridanus & conejo \\
\hline
\end{tabular}

\section{Generalidades conceptuales de la revisión}

Según MURPHY y LUGO (1986), el Bosque seco Tropical representaba el 50\% de las áreas boscosas en Centroamérica y el $22 \%$ en Sudamérica. En Colombia, según IAVH (1998), el Bosque seco Tropical se distribuía originalmente en las regiones de la llanura Caribe y valles interandinos de los ríos Magdalena y Cauca entre los 0 y $1000 \mathrm{~m}$ de altitud y en jurisdicción de los departamentos del Valle del Cauca, Tolima, Huila, Cundinamarca, Antioquía, Sucre, Bolívar, Cesar, Magdalena, Atlántico y sur de la Guajira. Según cálculos de este trabajo dentro de los $141 \mathrm{~km}^{2}$ de extensión con que cuenta el municipio de Colosó, los Montes de María, formación de Bosque seco Tropical típicamente estructurado por factores climáticos de sequía y presencia de suelos calcáreos, representa entre 40 y $45 \%$ del territorio del municipio de Colosó, Sucre; con referencia a este tipo de formación bioclimática son el último remanente que permanece casi inalterado dentro del departamento de Sucre y el país.

Se pudo establecer mediante la revisión de literatura para el área de estudio y extensible a los Montes de María, el registro de 7 familias y 13 especies de peces, 10 familias y 36 especies de anfibios, 14 familias y 52 especies de reptiles, 39 familias y 137 especies de aves, y 23 familias y 63 especies de mamíferos.

Al igual que los demás componentes de la diversidad biológica especifica, los vertebrados del Bosque seco Tropical, según CEBALLOS (1995), se pueden 
identificar como un grupo proveniente de los bosques húmedos y muy húmedos tropicales, que de forma adyacente se comunicaban con este tipo de ambiente a través de la continuidad ecosistémica o por medio de los corredores bióticos existentes.

En cuanto a la avifauna, se puede decir que existe una mezcla de elementos de zonas áridas y húmedas, por lo que posee un número reducido de aves especialistas, son en esencia de hábitos generalistas, se considera que las comunidades de aves ocupan un ecosistema de transición dentro de un gradiente climático (HAFFER, 1967; STOTZ et al., 1996).

Gran parte de la riqueza de vertebrados del Bosque seco Tropical depende directamente de la presencia de bosque húmedos y riparios que se encuentren cercanos dado a que las especies migran durante las épocas de sequía (CEBALLOS, 1995). En Colombia el Bosque seco Tropical se conecta hídricamente con ciénagas como en la región Caribe y con bosques húmedos circundantes como en el pie de monte de la Sierra Nevada de Santa Marta, en la Cordillera de los Andes y en el Magdalena Medio. Es probable, como lo sugiere IAVH (1998), que en el pasado haya existido una fauna rica asociada al Bosque seco Tropical y que el aislamiento y la fragmentación de los remanentes boscosos haya contribuido a que procesos biológicos como las migraciones estén en peligro o hayan desaparecido y con esto se haya reducido el tamaño de la poblaciones silvestres en función del tiempo y de la magnitud creciente de los impactos ambientales negativos, llegando incluso a advertirse posible extinción local.

Para el Caribe colombiano según IAVH (1997), particularmente para cuatro remanentes de Bosque seco Tropical (Zambrano, Los Colorado, Tierra Bomba y Neguanje), se registran 131 especies de aves con hábitos terrestres pertenecientes a 36 familias. De acuerdo con IAVH (1998), que a su vez se fundamenta en los listados de la literatura disponible sobre aves de Bosque seco en el Caribe colombiano (MARINKELLE, 1970; LUEPKE, 1972; MORENO, 1979; SERNA, 1984; MORALES, 1984; FORESTAL MONTERREY, 
1984; ANDRADE y MEJÍA, 1988) hay tres especies de aves que utilizan el bosque seco como hábitat primario Crypturellus erythropus, Accipiter bicolor e Icterus mesomelas que no han sido registradas dentro de áreas de bosque seco. Esta información sugiere la necesidad buscar registros de estas especies con la finalidad recomendar las áreas donde se encuentren como unidades de conservación.

IAVH (1998), sostiene que para los otros grupos de vertebrados en Colombia existen pocos estudios de composición y no existen trabajos comparativos entre regiones. CASTRO y KATTAN (1991); registran para el valle geográfico del río Cauca tres especies de anfibios, 21 de serpientes, 13 de saurios y una de quelonio. MORENO (1979), registra para el Parque Nacional Tayrona (Magdalena) en la región Caribe y en donde existen zonas de bosque húmedo, seco y matorrales subxerofíticos, 13 especies de anfibios, 14 de reptiles, 21 de murciélagos, 3 de primates, 2 de felinos, 5 de roedores y 2 especies de Edentata.

\section{Agradecimientos}

Por su apoyo a la Universidad de Sucre. A la Corporación Autónoma Regional de Sucre-CARSUCRE por la forma desinteresada con que brindó su acervo científico y permitió la consulta y utilización de su material escrito. A la Corporación ANP, por la oportunidad de compartir con sus profesionales las fases de campo llevadas a cabo.

\section{Referencias}

AGUILERA, M. 2005. La economía del departamento de Sucre: Ganadería y sector público. Documentos de trabajo sobre economía regional. Banco de la República N 63, Bogotá.

ANDRADE, G.; MEJIA, C. 1988. Cambios estacionales en la distribución de la avifauna terrestre en el parque nacional natural Macuira, Guajira. Trianea 1: 145-169. 
BARBOSA, C.; FAJARDO-PATIÑO, A.; GIRALDO, H.; RODRÍGUEZ, J. 1988. Proyecto sobre Evaluación del hábitat y Status del mono tití de cabeza blanca, Saguinus oedipus en Colombia. Unpublished Final Report of Status of Cottontop Tamarin in Colombia Project, INDERENA, Bogotá.

BELTRÁN, E. 1966. La administración de la fauna silvestre en Problemas de caza y pesca deportivas en México. Instituto Mexicano de Recursos Naturales Renovables, México.

CASTAÑO-MORA, O. (ed.). 2002. Libro Rojo de Reptiles de Colombia. Libros Rojos de Especies Amenazadas de Colombia. Instituto de Ciencias NaturalesUniversidad Nacional de Colombia, Ministerio del medio Ambiente, Conservación Internacional-Colombia. Bogotá.

CASTRO, F.; KATAN, G. 1991. Estado de conocimiento y conservación de los anfibio del Valle del Cauca. En Memorias primer simposio nacional de fauna del Valle del Cauca. CVC, Gobernación del Valle del Cauca, INCIVA. Cali.

CEBALLOS, G.; BULLOCK, S.; MEDINA, E.; MOONEY, H. (eds). 1995. Vertebrate diversity, ecology, and conservation in neotropical dry forest. En Tropical deciduous Forest Ecosystem.. Cambridge Univ. Press, Cambridge.

CUERVO, A.; BARBOSA, C.; DE LA OSSA, J. 1986. Aspectos ecológicos y etológicos de primates con énfasis en Alouatta seniculus (Cebidae), de la región de Colosó, Serranía de San Jacinto (Sucre), Costa Norte de Colombia. Caldasia vol. XIV, Nos. 68-70.

DAHL, G. 1971. Los peces del norte de Colombia. INDRENA, Bogotá.

DE LA OSSA, J.; RIAÑO, R.1997. Planificación y auditoría ambiental, Algunos conceptos estratégicos para manejar, usar y preservar los recursos naturales renovables. Corporación Autónoma Regional de Sucre y Sociedad Ambiental LTDA SOAM. Sucre.

EISENBERG, J.1989. Mammals of the Neotropics. The Northern neotropics.. The University of Chicago Press. Chicago.

FORESTAL MONTERREY. 1984. Avifauna de la región de Monterrey y Zambrano (Bolivar) - Aves de formaciones boscosas. Informe III. Estación Monterrey Forestal, Unidad de Investigación Biológica, Zambrano.

FROST, D. (ed.). 1985. Amphibiam species of the world. A taxonomic and geographical reference. Allen Press, Inc. \& The Association of Systematic Collections Lawrence, Kansas. 
GILES, R. 1978. Wildlife Management. W.H. Freeman Company, San Francisco.

GONDELLES, R.; MEDINA, G.; MÉNDEZ, J.; RIVERO BLANCO, C. 1981. Nuestros animales de caza. Guía para su conservación. Fundación de Educación Ambiental, MARNR, Caracas.

HAFFER, J. 1967. Zoogeographical notes on the "nonforest" lowland bird faunas of northwestern South America. Hornero, $10: 315-333$.

HILTY, S.; BROWN, W. 1986. A guide to the Birds of Colombia. Princeton University Press. Nueva Jersey.

IGAC. 1969. Monografía del departamento de Sucre. Oficina de Estudios Geográficos. Bogotá.

INSTITUTO ALEXANDER VON HUMBOLDT, IAVH. 1997. Caracterización ecológica de cuatro remanentes de Bosque seco Tropical de la región Caribe colombiana. Grupo de Exploraciones Ecologicas Rapidas, IAVH, Villa de Leyva.

INSTITUTO ALEXANDER VON HUMBOLDT, IAVH. 1998. EI Bosque seco Tropical en Colombia. Grupo de Exploraciones Ecologicas Rapidas, IAVH, Villa de Leyva.

LUEPKE, J. 1972. Descripción de algunas especies de aves del parque nacional Tay rona. INDERENA.

MARINKELLE, C. 1970. Birds of the serrania de Macuira, Guajira peninsula Colombia. Mitt. Inst. Colombo-Alemán Invest. Cient. 4:15-34.

MINISTERIO DEL AMBIENTE. 2002. Plan Nacional en Bioprospección Continental y marina. Bogotá.

MINISTÉRIO DO MEIO AMBIENTE MMA - DCBio. 2004. Estrategias Nacionales de Biodiversidad en América del Sur: Perspectivas para la Cooperación Regional. Brasilia.

MORENO, L. 1979. Especies de vertebrados que han sido identificadas en el Parque Nacional Natural Tayrona en los diferentes muestreos realizados. INDERENA, Documento Interno. 
MORALES, J.1984. Informe sobre la avifauna del parque nacional natural Tayrona. II Expedición Botánica. INDERENA, Documento interno.

MUÑOZ-ARANGO, J. 2001. Los Murciélagos de Colombia. Sistemática, distribución, descripción, historia natural y ecología. Editorial Universidad de Antioquia. Medellín.

MURPHY, P; LUGO, A. 1986. Ecology of tropical dry forest. Annals Review of Ecology and Systematics $17: 67-68$.

MCNEELY, J. 1988. Economics and biological diversity. UICN, Gland, Suiza.

OJASTI, J. 2000. Manejo de Fauna Silvestre Neotropical Instituto de Zoologia Tropical Universidad Central de Venezuela, Caracas.

OVIEDO, U. 2008. The Montes de María: a territory of contrasts. Natural wealth in front of social poverty. Revista BÚSQUEDA. CECAR. 9:110-129.

ONU. NACIONES UNIDAS. 1994. Contabilidad ambiental y económica integrada. ST/ESA/STAT/ SER.F/61. Naciones Unidas, NuevaYork.

PRIMACK, R. 1998. Essentials of Conservation Biology. Sinauer Associates. Publishers. Sunderland, Masachusetts.

PROYECTO DE PROTECCIÓN INTEGRAL DE AGUAS SUBTERRÁNEAS "PPIAS" Acuífero Morroa. 2005. CARSUCRE. Documento Interno Corporación Autónoma Regional de Sucre.

RODRÍGUEZ-MAHECHA, J.; ALBERICO, M.; TRUJILLO, F.; JORGENSON, J. (eds.). 2006. Libro Rojo de Mamíferos de Colombia. Serie Libros Rojos de Especies Amenazadas de Colombia. Conservación Internacional-Colombia, Instituto de Ciencias Naturales-Universidad Nacional de Colombia, Ministerio del medio Ambiente, Bogotá.

RUEDA-ALMONACID, J.; LYNCH, J.; AMÉSQUITA, A. (eds.). 2004. Libro Rojo de Anfibios de Colombia. Serie Libros Rojos de Especies Amenazadas de Colombia. Conservación Internacional-Colombia, Instituto de Ciencias NaturalesUniversidad Nacional de Colombia, Ministerio del medio Ambiente, Bogotá.

RUIZ, P.; ARDILA, V.; LYNCH, J. 1996. Lista actualizada de la fauna amphibia de Colombia. Rev. Acad. Colomb. Cienc. Vol. XX No 77. 
SÁNCHEZ, H.; CASTAÑO, O.; CÁRDENAS, G. 1992. Diversidad de los Reptiles en Colombia. En: Colombia Diversidad Biótica I. Editor: Orlando Rangel. Instituto de Ciencias Naturales, Universidad Nacional de Colombia. Bogotá.

SERNA, M. 1984. Avifauna parcial de la Guajira. Colegio San José. Medellin.

SCHUERHOLZ, G.; MANN, G. 1979. Proposición para la administración y el manejo de los recursos de vida silvestre en Chile. FAO DP/CHI/76/003, Santiago.

STOTZ, D.; FITZPATRICK, J.; PARKER III, T.; MOSKOVITS, D. 1996. Neotropical birds - Ecolygy and conservation. The University Chicago Press, Chicago.

USHER, M. (ed.). 1986. Wildlife conservation education. Chapmann and Hall, Londres.

UNEP. 1993. Guidelines for country studies on biological diversity. United Nations Environment Programme, Nairobi.

WING, L. 1951. Practice of wildlife conservation. John Wiley \& Sons, Nueva York. 